\title{
BOUNDS ON VOLUME GROWTH OF GEODESIC BALLS UNDER RICCI FLOW
}

\author{
QI S. ZHANG
}

\begin{abstract}
We prove a so-called $\kappa$ non-inflating property for Ricci flow, which provides an upper bound for volume ratio of geodesic balls over Euclidean ones, under an upper bound for scalar curvature. This result can be regarded as the opposite statement of Perelman's $\kappa$ non-collapsing property for Ricci flow. These two results together imply volume-doubling property for Ricci flow without assuming Ricci curvature lower bound.
\end{abstract}

\section{Statement of result and proof}

In $[\mathrm{P}]$, Perelman proved the fundamental $\kappa$ non-collapsing property for Ricci flow. One version of it roughly says that the volume ratio between a geodesic ball and Euclidean ball with the same radius is bounded from below by a positive constant, provided that the scalar curvature is bounded from above in a space-time cube.

In this short note, we prove that the opposite result is also true. i.e., the above volume ratio is bounded from above by a positive constant, provided that the scalar curvature is bounded from above in a space-time cube. In the case of normalized Ricci flow on compact Kähler manifolds with positive first Chern class, the upper bound holds for all time. An upper bound for the volume ratio is useful in the study of Kähler-Ricci flow. See, for example, the papers [Se, CW] and the references therein. The current result seems to remove one of the obstacles in the program to prove convergence results, although many other obstacles remain.

To make the statement precise, let us introduce notations and definition. We use $\mathbf{M}$ to denote a compact Riemann manifold and $g(t)$ to denote the metric at time $t$; $d(x, y, t)$ is the geodesic distance under $g(t) ; B(x, r, t)=\{y \in \mathbf{M} \mid d(x, y, t)<r\}$ is the geodesic ball of radius $r$, under metric $g(t)$, centered at $x$, and $|B(x, r, t)|_{g(t)}$ is the volume of $B(x, r, t)$ under $g(t) ; d \mu_{g(t)}(x)$ is the volume element. We also reserve $R=R(x, t)$ as the scalar curvature under $g(t)$.

Definition 1.1. A smooth, compact, $n$-dimensional Ricci flow $(\mathbf{M}, g(t))$ is called $\kappa$ non-inflated at the point $\left(x_{0}, t_{0}\right)$ under scale $\rho$ if the following statement holds.

For any $r \in(0, \rho)$, suppose:

1. The Ricci flow is defined in the space-time cube

$$
\left\{(x, t) \mid d\left(x, x_{0}, t_{0}\right)<r, \quad t \in\left[t_{0}-r^{2}, t_{0}\right]\right\} .
$$

2. For some positive constant $\alpha, R(x, t) \leq \frac{\alpha}{t_{0}-t}$ for all $(x, t)$ in the above cube. Then, there exists a positive constant $\kappa$, which may depend on $\alpha$ such that

$$
\left|B\left(x_{0}, r, t_{0}\right)\right|_{g\left(t_{0}\right)} \leq \kappa r^{n} .
$$

Received by the editors July 4, 2011. 
Remark. Recall that in the $\kappa$ non-collapsing property, the condition on the scalar curvature is $R(x, t) \leq \frac{1}{r^{2}}$ in the space-time cube. Obviously this condition is included in our condition $R(x, t) \leq \frac{\alpha}{t_{0}-t}$ in the same space-time cube.

The main result of the note is the following theorem. Even though the proof is very short, it actually uses a combination of several results: a variation of global bounds for the fundamental solution of the conjugate heat equation ([CZ]) whose proof relies on Perelman's Harnack inequality in $[\mathrm{P}]$ and uniform Sobolev inequality under Ricci flow, and also Perelman's scalar curvature and diameter bound for Kähler-Ricci flow, and the general idea that heat kernel lower bound implies volume upper bound in [GHL].

Theorem 1.1. (a). Let $(\mathbf{M}, g(t)), \partial_{t} g_{i j}=-2 R_{i j}, t \in\left[0, t_{0}\right]$ be a smooth, compact, $n$-dimensional Ricci flow. Then for any $x_{0} \in \mathbf{M}$, the Ricci flow is $\kappa$ non-inflated at $\left(x_{0}, t_{0}\right)$ under scale $\sqrt{t_{0}}$. Here $\kappa$ depends only on $g(0), t_{0}$ and the constant $\alpha$ in the bound for the scalar curvature.

(b). Let $(\mathbf{M}, g(t)), \partial_{t} g_{i j}=-R_{i j}+g_{i j}, t \in[0, \infty)$ be a smooth, compact, $n$-real dimensional, normalized Ricci flow on compact Kähler manifolds with positive first Chern class. There exists a positive constant $\kappa>0$, which depends only on the initial metric $g(0)$ such that

$$
|B(x, r, t)| \leq \kappa r^{n}
$$

for all $x \in \mathbf{M}, r>0$ and $t>0$.

Proof. (of part (a)).

Step 1 .

Picking any $r \in\left(0, \sqrt{t_{0}}\right)$, we assume:

1. The Ricci flow is defined in the space-time cube

$$
Q\left(x_{0}, t_{0}, r\right)=\left\{(x, t) \mid d\left(x, x_{0}, t_{0}\right)<r, \quad t \in\left[t_{0}-r^{2}, t_{0}\right]\right\} .
$$

2. For some positive constant $\alpha, R(x, t) \leq \frac{\alpha}{t_{0}-t}$ for all $(x, t)$ in the above cube $Q\left(x_{0}, t_{0}, r\right)$.

Let $l$ and $t$ be two moments in time such that $0<l<t \leq t_{0}$ and $x, z \in \mathbf{M}$. Let $G=G(z, l ; x, t)$ be the fundamental solution of the conjugate heat equation

$$
\Delta u-R u+\partial_{l} u=0,
$$

which is coupled with the Ricci flow. Fixing $z, l$, we know that $G$, as a function of $x, t$ satisfies the heat equation i.e., for $t>l$,

$$
\Delta_{x} G(z, l ; x, t)-\partial_{t} G(z, l ; x, t)=0 .
$$

Hence,

$$
\begin{aligned}
\frac{d}{d t} \int_{\mathbf{M}} G(z, l ; x, t) d \mu_{g(t)}(x) & =\int_{\mathbf{M}}\left[\Delta_{x} G(z, l ; x, t)-R(x, t) G(z, l ; x, t)\right] d \mu_{g(t)}(x) \\
& =-\int_{\mathbf{M}} R(x, t) G(z, l ; x, t) d \mu_{g(t)}(x) .
\end{aligned}
$$

From the scalar curvature equation

$$
\Delta R+2|R i c|^{2}-\partial_{t} R=0
$$


we deduce

$$
\Delta R+\frac{2}{n} R^{2}-\partial_{t} R \leq 0
$$

which implies, via the maximum principle that, either $R(\cdot, 0) \geq 0$ or

$$
\min R(\cdot, t) \geq \frac{1}{(1 / \min R(\cdot, 0))-(2 t / n)} .
$$

Therefore, either $\frac{d}{d t} \int_{\mathbf{M}} G(z, l ; x, t) d \mu_{g(t)}(x) \leq 0$ or

$$
\frac{d}{d t} \int_{\mathbf{M}} G(z, l ; x, t) d \mu_{g(t)}(x) \leq \frac{1}{(-1 / \min R(\cdot, 0))+(2 t / n)} \int_{\mathbf{M}} G(z, l ; x, t) d \mu_{g(t)}(x),
$$

which yields

$$
\int_{\mathbf{M}} G(z, l ; x, t) d \mu_{g(t)}(x) \leq 1+C(1+t-l)^{n / 2} .
$$

Here $C$ only depends on $\min R(\cdot, 0)$ and $n$, and $C=0$ when $R \geq 0$.

\section{Step 2.}

Next, we prove the following heat kernel bounds on $G(z, l ; x, t)$ which is similar to Theorem 2.1 in [CZ]. The method is also similar. The improvement is on the coefficients of the bounds, which rely on the initial metric $g(0)$ instead of on $g(l)$. This will be useful in proving the volume ratio bound.

$$
\frac{c_{1} J(t)}{(t-l)^{n / 2}} e^{-2 c_{2} \frac{d(z, x, t)^{2}}{t-l}} e^{-\frac{1}{\sqrt{t-l}} \int_{l}^{t} \sqrt{t-s} R(x, s) d s} \leq G(z, l ; x, t) \leq \frac{c_{1}^{-1} J^{-1}(t)}{(t-l)^{n / 2}} .
$$

Here

$$
J=J(s)=\exp \left[-\alpha-s \beta-s \sup R^{-}(\cdot, 0)\right],
$$

and $\alpha$ and $\beta$ are positive constants depending only on the Sobolev constants of $(\mathbf{M}, g(0))$ and the infimum of Perelman's $F$ entropy for $(\mathbf{M}, g(0))$. The proof of this theorem uses uniform Sobolev inequality under Ricci flow and Perelman's differential Harnack inequality for $G[\mathrm{P}]$. Moreover, if the scalar curvature is positive, then $J(t)$ is independent of $t$.

Recall that as a function of $(x, t), G=G(z, l ; x, t)$ is the fundamental solution of the forward heat equation associated with the Ricci flow, i.e.,

$$
\left\{\begin{array}{l}
\frac{\partial}{\partial t} g(t)=-2 R i c \\
u_{t}=\Delta u
\end{array}\right.
$$

First, we study the forward heat equation (1.4).

Let $u=u(x, t)$ be a positive solution to (1.4). Given $T>0$ and $t \in(l, T)$, define

$$
p(t)=(T-l) /(T-t),
$$


so $p(l)=1$ and $p(T)=\infty$. By direct computation, using the idea of Davies [D],

$$
\begin{aligned}
\partial_{t}\|u\|_{p(t)}= & \partial_{t}\left[\left(\int_{\mathbf{M}} u^{p(t)}(x, t) d \mu_{g(t)}\right)^{1 / p(t)}\right] \\
= & -\frac{p^{\prime}(t)}{p^{2}(t)}\|u\|_{p(t)} \ln \int_{\mathbf{M}} u^{p(t)}(x, t) d \mu_{g(t)} \\
& +\frac{1}{p(t)}\left(\int_{\mathbf{M}} u^{p(t)}(x, t) d \mu_{g(t)}\right)^{(1 / p(t))-1} \\
& \times\left[\int_{\mathbf{M}} u^{p(t)}(\ln u) p^{\prime}(t) d \mu_{g(t)}+\int_{\mathbf{M}} u^{p(t)-1}(p(t) \Delta u-R u) d \mu_{g(t)}\right] .
\end{aligned}
$$

Using integration by parts on the term containing $\Delta u$ and multiplying both sides by $p^{2}(t)\|u\|_{p(t)}^{p(t)}$, we infer

$$
\begin{aligned}
p^{2}(t) & \|u\|_{p(t)}^{p(t)} \partial_{t}\|u\|_{p(t)} \\
= & -p^{\prime}(t)\|u\|_{p(t)}^{p(t)+1} \ln \int_{\mathbf{M}} u^{p(t)}(x, t) d \mu_{g(t)}+p(t)\|u\|_{p(t)} p^{\prime}(t) \int_{\mathbf{M}} u^{p(t)} \ln u(x, t) d \mu_{g(t)} \\
& -p^{2}(t)(p(t)-1)\|u\|_{p(t)} \int_{\mathbf{M}} u^{p(t)-2}|\nabla u|^{2}(x, t) d \mu_{g(t)} \\
& -p(t)\|u\|_{p(t)} \int_{\mathbf{M}} R(x, t) u^{p(t)}(x, t) d \mu_{g(t)} .
\end{aligned}
$$

Dividing both sides by $\|u\|_{p(t)}$, we arrive at

$$
\begin{aligned}
p^{2}(t)\|u\|_{p(t)}^{p(t)} \partial_{t} \ln \|u\|_{p(t)} & \\
= & -p^{\prime}(t)\|u\|_{p(t)}^{p(t)} \ln \int_{\mathbf{M}} u^{p(t)} d \mu_{g(t)}+p(t) p^{\prime}(t) \int_{\mathbf{M}} u^{p(t)} \ln u d \mu_{g(t)} \\
& -4[p(t)-1] \int_{\mathbf{M}}\left|\nabla\left(u^{p(t) / 2}\right)\right|^{2} d \mu_{g(t)}-p(t) \int_{\mathbf{M}} R\left(u^{p(t) / 2}\right)^{2} d \mu_{g(t)}
\end{aligned}
$$

Define $v(x, t)=\frac{u^{p(t) / 2}}{\left\|u^{p(t) / 2}\right\|_{2}}$, then $\|v\|_{2}=1$ and

$$
v^{2} \ln v^{2}=p(t) v^{2} \ln u-2 v^{2} \ln \left\|u^{p(t) / 2}\right\|_{2} .
$$

Merging the first two terms on the right-hand side of the above equality and dividing both sides by $\|u\|_{p(t)}^{p(t)}$, we find that

$$
\begin{aligned}
& p^{2}(t) \partial_{t} \ln \|u\|_{p(t)} \\
& \quad=p^{\prime}(t) \int_{\mathbf{M}} v^{2} \ln v^{2} d \mu_{g(t)}-4(p(t)-1) \int_{\mathbf{M}}|\nabla v|^{2} d \mu_{g(t)}-p(t) \int_{\mathbf{M}} R v^{2} d \mu_{g(t)} \\
& \quad=p^{\prime}(t) \int_{\mathbf{M}} v^{2} \ln v^{2} d \mu_{g(t)}-4[p(t)-1] \int_{\mathbf{M}}\left(|\nabla v|^{2}+\frac{1}{4} R v^{2}\right) d \mu_{g(t)}-\int_{\mathbf{M}} R v^{2} d \mu_{g(t)} .
\end{aligned}
$$

Note the following relations:

$$
\frac{4(p(t)-1)}{p^{\prime}(t)}=\frac{4(t-l)(T-l-(t-l))}{T-l} \leq T-l, \quad \frac{1}{p^{\prime}(t)}=\frac{(T-l)^{2}}{T} \leq T,
$$


Hence,

$$
\begin{aligned}
p^{2}(t) \partial_{t} \ln \|u\|_{p(t)} \leq & p^{\prime}(t)\left[\int_{\mathbf{M}} v^{2} \ln v^{2} d \mu_{g(t)}-\frac{4(p(t)-1)}{p^{\prime}(t)}\right. \\
& \left.\times \int_{\mathbf{M}}\left(|\nabla v|^{2}+\frac{1}{4} R v^{2}\right) d \mu_{g(t)}+T \sup R^{-}(\cdot, t)\right] .
\end{aligned}
$$

Next, we recall the log-Sobolev inequality (6.2.8) in Section 6.2 of [Z2]

$$
\begin{aligned}
\int_{\mathbf{M}} v^{2} \ln v^{2} d \mu_{g(t) \leq} & \epsilon^{2} \int_{\mathbf{M}}\left(4|\nabla v|^{2}+R v^{2}\right) d \mu_{g(t)}-n \ln \epsilon+\left(t+\epsilon^{2}\right) B A^{-1} \\
& +n 2^{-1} \ln \left(n A 2^{-1}\right)-n 2^{-1} .
\end{aligned}
$$

Here $A$ and $B$ only depend on the Sobolev constants and the infimum of the $F$ entropy of the initial metric. Taking $\epsilon$ such that

$$
\epsilon^{2}=\frac{4(p(t)-1)}{p^{\prime}(t)} \leq T-l
$$

in the above inequality, we deduce that

$$
p^{2}(t) \partial_{t} \ln \|u\|_{p(t)} \leq p^{\prime}(t)\left[-n \ln \sqrt{4(p(t)-1) / p^{\prime}(t)}+L(t)+T \sup R^{-}(\cdot, 0)\right],
$$

where, due to $\epsilon^{2} \leq T-l \leq T$,

$$
\begin{aligned}
L(t) & \doteq\left(t+\epsilon^{2}\right) \beta+\alpha \\
& \leq 2 T \beta+\alpha \\
& \doteq L(T),
\end{aligned}
$$

for some positive constants $\alpha=\alpha\left(A_{0}, B_{0}, \lambda_{0}, n\right)$ and $\beta=\beta\left(A_{0}, B_{0}, \lambda_{0}, n\right)$. Here $A_{0}$ and $B_{0}$ are the coefficients in the standard Sobolev inequality for $(\mathbf{M}, g(0))$ and $\lambda_{0}$ is the infimum of Perelman's $F$ entropy for $(\mathbf{M}, g(0))$. We stress that these depend only on initial metric. Here, we also used the fact that

$$
\sup R^{-}(x, t) \leq \sup R^{-}(x, 0)
$$

which is a consequence of maximum principle and the evolution equation of scalar curvature $\partial_{t} R=\Delta R+2|R i c|^{2}$.

Observe that $p^{\prime}(t) / p^{2}(t)=1 /(T-l)$ and

$$
4(p(t)-1) / p^{\prime}(t)=4(t-l)[T-l-(t-l)] /(T-l) .
$$

Hence, we have

$$
\begin{aligned}
& \partial_{t} \ln \|u\|_{p(t)} \\
& \quad \leq \frac{1}{T-l}\left\{-\frac{n}{2} \ln [4(t-l)[T-l-(t-l)] /(T-l)]+L(T)+T \sup R^{-}(\cdot, 0)\right\} .
\end{aligned}
$$

This implies, after integrating from $t=l$ to $t=T$, that

$$
\ln \frac{\|u(\cdot, T)\|_{\infty}}{\|u(\cdot, l)\|_{1}} \leq-\frac{n}{2} \ln (4(T-l))+L(T)+T \sup R^{-}(\cdot, 0)+n .
$$

Since

$$
u(x, T)=\int_{\mathbf{M}} G(z, l ; x, T) u(z, l) d \mu_{g(l)},
$$


the above inequality implies that

$$
G(z, l, x, T) \leq \frac{\exp \left[L(T)+T \sup R^{-}(\cdot, 0)\right]}{(4(T-l))^{n / 2}},
$$

where $L(T)$ is defined above as

$$
L(T)=2 T \beta+\alpha .
$$

Since $T$ is an arbitrary, we get the upper bound by defining $J^{-1}=L(T)+T$ sup $R^{-}(\cdot, 0)$. Note the constants $\beta$ and $\alpha$ may have changed by a factor.

In case $R(x, 0)>0$, by Section 6.2 in [Z2], we have $\beta=0$. So the above bound becomes

$$
G(z, l ; x, T) \leq \frac{\exp (\alpha)}{(4 \pi(T-l))^{n / 2}},
$$

proving the upper bound.

Next, we prove the lower bound. Let $t<t_{0}$ and $u=u(x, t) \equiv G\left(x, t ; x_{0}, t_{0}\right)$. We claim that for a constant $C>0$,

$$
G\left(x_{0}, t ; x_{0}, t_{0}\right) \geq \frac{C}{\tau^{n / 2}} e^{-\frac{1}{2 \sqrt{\tau}} \int_{t}^{t_{0}} \sqrt{t_{0}-s} R\left(x_{0}, s\right) d s},
$$

where $\tau=t_{0}-t$ here and later in the proof. To prove this inequality, define a function $f$ by

$$
(4 \pi \tau)^{-n / 2} e^{-f}=u \text {. }
$$

We need to apply Perelman's differential Harnack inequality for the fundamental solution along any smooth curve $\gamma(t)$ (see [P, Corollary 9.4]). Here we pick the curve $\gamma(t)$ to be the fixed point $x_{0}$, we have,

$$
-\partial_{t} f\left(x_{0}, t\right) \leq \frac{1}{2} R\left(x_{0}, t\right)-\frac{1}{2 \tau} f\left(x_{0}, t\right) .
$$

For any $t_{2}<t_{1}<t_{0}$, we integrate the above inequality to get

$$
f\left(x_{0}, t_{2}\right) \sqrt{t_{0}-t_{2}} \leq f\left(x_{0}, t_{1}\right) \sqrt{t_{0}-t_{1}}+\frac{1}{2} \int_{t_{2}}^{t_{1}} \sqrt{t_{0}-s} R\left(x_{0}, s\right) d s .
$$

When $t_{1}$ approaches $t_{0}, f\left(x_{0}, t_{1}\right)$ stays bounded since $G\left(x_{0}, t_{1} ; x_{0}, t_{0}\right)\left(t_{0}-t_{1}\right)^{n / 2}$ is bounded between two positive constants, which is a direct consequence of the standard asymptotic formula for $G$ (for example, see [C++, Chapter 24, p. 278]). Hence for any $t \leq t_{0}$, we have

$$
f\left(x_{0}, t\right) \leq \frac{1}{2 \sqrt{t_{0}-t}} \int_{t}^{t_{0}} \sqrt{t_{0}-s} R\left(x_{0}, s\right) d s
$$

Consequently,

$$
G\left(x_{0}, t ; x_{0}, t_{0}\right) \geq \frac{c}{(4 \pi \tau)^{n / 2}} e^{-\frac{1}{2 \sqrt{t_{0}-t}} \int_{t}^{t_{0}} \sqrt{t_{0}-s} R\left(x_{0}, s\right) d s} .
$$

As observed earlier, the function $G\left(x_{0}, t ; \cdot, \cdot\right)$ is a solution to the standard heat equation coupled with Ricci flow, which is the conjugate of the conjugate heat equation. i.e.,

$$
\Delta_{z} G(x, t ; z ; l)-\partial_{l} G(x, t ; z, l)=0
$$


here $\Delta_{z}$ is with respect to the metric $g(l)$. Therefore, it follows from [Z1, Theorem 3.3] or [CH, Theorem 5.1] that, for $\delta>0, c_{1}, c_{2}>0$, and $y_{0} \in \mathbf{M}$,

$$
G\left(x_{0}, t ; x_{0}, t_{0}\right) \leq c_{1} G^{1 /(1+\delta)}\left(x_{0}, t, y_{0}, t_{0}\right) K^{\delta /(1+\delta)} e^{c_{2} d^{2}\left(x_{0}, y_{0}, t_{0}\right) / \tau},
$$

where $K=\sup _{M \times\left[\left(t_{0}+t\right) / 2, t_{0}\right]} G\left(x_{0}, t, \cdot, \cdot\right)$. The upper bound

$$
K \leq \frac{c J^{-1}\left(t_{0}\right)}{\left(t_{0}-t\right)^{n / 2}}
$$

together with the lower bound (1.7) imply that, with $\delta=1$,

$$
G\left(x_{0}, t ; y_{0}, t_{0}\right) \geq c_{1} \frac{J\left(t_{0}\right)}{\left(t_{0}-t\right)^{n / 2}} e^{-2 c_{2} d\left(x_{0}, y_{0}, t_{0}\right)^{2} / \tau} e^{-\frac{1}{\sqrt{t_{0}-t}} \int_{t}^{t_{0}} \sqrt{t_{0}-s} R\left(x_{0}, s\right) d s},
$$

which is the desired lower bound.

\section{Step 3.}

In (1.3), we take $z=x_{0}, t=t_{0}$ and $l=t_{0}-r^{2}$, where $r$ is the given number in $\left(0, \sqrt{t_{0}}\right)$. By the assumption on the scalar curvature $R(x, t)$ in the definition of $\kappa$ non-inflating, we obtain, for $x$ such that $d\left(x_{0}, x, t_{0}\right) \leq r$,

$$
\begin{aligned}
G\left(x_{0}, t_{0}-r^{2} ; x, t_{0}\right) & \geq \frac{c_{1} J\left(t_{0}\right)}{r^{n}} e^{-2 c_{2}} e^{-\frac{1}{r} \int_{t_{0}-r^{2}}^{t_{0}} \sqrt{t_{0}-s} R(x, s) d s} \\
& \geq \frac{c_{1} J\left(t_{0}\right)}{r^{n}} e^{-2 c_{2}} e^{-\frac{1}{r} \int_{t_{0}-r^{2}}^{t_{0}} \sqrt{t_{0}-s} \frac{\alpha}{t_{0}-s} d s} .
\end{aligned}
$$

Thus, when $d\left(x_{0}, x, t_{0}\right) \leq r$, we have

$$
G\left(x_{0}, t_{0}-r^{2} ; x, t_{0}\right) \geq \frac{c_{1} J\left(t_{0}\right)}{r^{n}} e^{-2 c_{2}-2 \alpha} .
$$

Substituting this into (1.2), we deduce

$$
\begin{aligned}
1+C\left(1+r^{2}\right)^{n / 2} & \geq \int_{\mathbf{M}} G\left(x_{0}, t_{0}-r^{2} ; x, t_{0}\right) d \mu_{g\left(t_{0}\right)}(x) \\
& \geq \int_{d\left(x_{0}, x, t_{0}\right) \leq r} G\left(x_{0}, t_{0}-r^{2} ; x, t_{0}\right) d \mu_{g\left(t_{0}\right)}(x) \\
& \geq \frac{c_{1} J\left(t_{0}\right)}{r^{n}} e^{-2 c_{2}-2 \alpha} \int_{d\left(x_{0}, x, t_{0}\right) \leq r} d \mu_{g\left(t_{0}\right)}(x) .
\end{aligned}
$$

This implies

$$
\left|B\left(x_{0}, r, t_{0}\right)\right|_{g\left(t_{0}\right)} r^{-n} \leq\left[1+C\left(1+t_{0}\right)^{n / 2}\right] e^{2 c_{2}+2 \alpha} c_{1}^{-1} J^{-1}\left(t_{0}\right) .
$$

Taking

we obtain

$$
\kappa=\left[1+C\left(1+t_{0}\right)^{n / 2}\right] e^{2 c_{2}+2 \alpha} c_{1}^{-1} J^{-1}\left(t_{0}\right),
$$

$$
\left|B\left(x_{0}, r, t_{0}\right)\right|_{g\left(t_{0}\right)} \leq \kappa r^{n}
$$

proving part (a) of the theorem. Note $\kappa$ depends only on $t_{0}$ and $g(0)$ in general, and if $R \geq 0$, then $\kappa$ only depends on $g(0)$, due to the aforementioned property on the constant $J$ in (1.3), and the fact that in the expression of $\kappa$, the constant $C=0$ when $R \geq 0$. 
Proof. (of part (b)). Since the normalized Ricci flow is smooth, we only need to prove the result for $t \geq c_{0}$ for some positive constant $c_{0}$. According to Perelman (see [ST]), the scalar curvature $R=R(x, t)$ and diameter of the manifold are uniformly bounded for all time. Using the scaling

$$
t=-\ln (1-2 \tilde{t}), \quad g(t)=\frac{1}{1-2 \tilde{t}} \tilde{g}(\tilde{t}),
$$

we see that $\tilde{g}(\tilde{t})$ is the standard Ricci flow in the time interval $\tilde{t} \in[0,1 / 2)$ such that

$$
\tilde{R}(x, \tilde{t}) \leq \frac{\alpha}{1-2 \tilde{t}},
$$

where $\alpha$ is a positive constant. Pick $\tilde{t} \in[1 / 4,1 / 2)$ and $\tilde{r} \in(0,1 / 2)$. Then for all $s \in\left[\tilde{t}-\tilde{r}^{2}, \tilde{t}\right]$ and $x \in \mathbf{M}$, we have

$$
\tilde{R}(x, s) \leq \frac{\alpha / 2}{(1 / 2)-s} \leq \frac{\alpha}{\tilde{t}-s} .
$$

Now we can just apply part (a) of the theorem to conclude

$$
|\{y \mid d(y, x, \tilde{g}(\tilde{t}))<\tilde{r}\}|_{\tilde{g}(\tilde{t})} \leq \kappa \tilde{r}^{n}
$$

where $\kappa$ depends only on the initial metric $g(0)$ and $\alpha$. This is so because the total length of time interval is $1 / 2$ for $\tilde{t}$. After scaling we obtain, for $r=(1-2 \tilde{t})^{-1 / 2} \tilde{r}=$ $e^{t / 2} \tilde{r}$,

$$
|B(x, r, t)|_{g(t)} \leq \kappa r^{n} .
$$

Since $\tilde{r}$ can be any number in $(0,1 / 2)$, we conclude that for $r \leq e^{t / 2} / 2$, and all $t \geq \ln 2$,

$$
|B(x, r, t)|_{g(t)} r^{-n} \leq \kappa .
$$

Since the diameter of $(\mathbf{M}, g(t))$ is uniformly bounded, the above holds for all $r>0$ with perhaps a different constant $\kappa$.

\section{Acknowledgments}

We wish to thank Professors Xiaodong Cao and Xiouxiong Chen for very helpful suggestions. After the paper is circulated and posted on the arXiv, Professor Xiouxiong Chen kindly informed us that he and Professor Bing Wang also independently obtained a similar result under the condition that scalar curvature has global upper and extra lower bound, which is just posted [CW2]. Thanks also go to Professor Hongliang Shao for checking the paper carefully and correcting a typo.

\section{References}

[CH] X. Cao and R.S. Hamilton, Differential Harnack estimates for time-dependent heat equations with potentials, Geom. Funct. Anal. 19(4) (2009), 989-1000.

$[\mathrm{C}++]$ B. Chow, S.-C. Chu, D. Glickenstein, C. Guenther, J. Isenberg, T. Ivey, D. Knopf, P. Lu, F. Luo and L. Ni, The Ricci flow: techniques and applications. Part III, Mathematical Surveys and Monographs, American Mathematical Society, Providence, RI, Geometric-Analysis Aspects, 2010, 517pp.

[CW] X. Chen and B. Wang, Space of Ricci flows (I), arXiv:0902.1545

[CW2] X. Chen and B. Wang, On the conditions to extend Ricci flow(III), arXiv:1107.5110 
[CZ] X. Cao and Q.S. Zhang, The conjugate heat equation and ancient solutions of the Ricci Flow, Math., Adv. Math. 228 (2011), 2891-2919, arXiv: 1006.0540.

[D] E.B. Davies, Explicit constants for Gaussian upper bounds on heat Kernels, Amer. J. Math. 109(2) (1987), 319-333.

[GHL] A. Grigor'yan, J. Hu and K.-S. Lau, Heat Kernels on metric-measure spaces and an application to semi-linear elliptic equations, Trans. AMS 355(5) (2003), 2065-2095.

[P] G. Perelman, The entropy formula for the Ricci flow and its geometric applications, Math. arXiv:math.DG/0211159.

[Se] N. Sesum, Convergence of a Kähler-Ricci flow, Math. Res. Lett. 12(5-6) (2005), 623-632.

[ST] N. Sesum and G. Tian, Bounding scalar curvature and diameter along the Kähler Ricci flow (after Perelman), J. Inst. Math. Jussieu 7(3) (2008), 575-587.

[Z1] Q.S. Zhang, Some gradient estimates for the heat equation on domains and for an equation by Perelman, Int. Math. Res. Not. pages Art. ID 92314, 2006 (2006), 1-39.

[Z2] Q.S. Zhang, Sobolev inequalities, heat kernels under Ricci flow and the Poincaré conjecture, CRC Press, Boca Raton, FL, 2011.

Department of Mathematics, University of California, Riverside, CA 92521, USA

E-mail address: qizhang@math.ucr.edu 
